including catechin are some pharmacologically active compounds found in relatively large amounts in chocolate. The relevant chemical agent responsible for initiating a migraine attack requires to be elucidated. My own clinical experience in children suggests that chocolate is a common precipitating factor in migraine susceptible patients. The effects of eliminating chocolate and caffeine containing drinks should be investigated before long-term drug treatment is considered.

\title{
BLOOD MAGNESIUM LEVELS IN MIGRAINE
}

Serum and erythrocyte magnesium levels were screened between attacks in patients with migraine without aura $(n=38)$ and with aura $(n=6)$ and for comparison, in a group of patients suffering from chronic tension-type headache $(n=25)$ as well as a group of control patients $(n=19)$ at the Headache Unit, Citadelle University of Liege, Liege, Belgium. Serum magnesium levels were not significantly different between the four groups of patients, whereas magnesium levels in erythrocytes were significantly reduced in the group of migraineurs without aura (1.87) compared with those with aura (2.1), patients with tension-type headache (2.03) and the controls (2.12). Normal values in the laboratory ranged from 2-2.8 $\mathrm{mmol} / \mathrm{l}$ in erythrocytes (Schoenen J. et al. Blood magnesium levels in migraine. Cephalagia May 1991; 11:97-99).

COMMENT. The correlation between magnesium levels and migraine pathophysiology is speculative. The possible importance of magnesium in migraine is suggested by the finding of reduced magnesium in white blood cells in women with premenstrual syndrome and headaches and the benefits of oral treatment with magnesium in premenstrual headaches. The authors hypothesize that the reduction in erythrocyte magnesium may be due to an abnormal regulation of intracellular magnesium in migraine patients.

\section{ESTROGENS, PROGESTINS, AND HEADACHE}

Approaches to the therapy of hormone-related headaches are reviewed from the Department of Neurology, Temple University School of Medicine and the Comprehensive Headache Center at Germantown Hospital, Philadelphia, PA, and the Reproductive Endocrine Unit, National Institute of Child Health and Human Development, NIH, Bethesda, MD. Migraine can occur before, during, or after menstruation, or at the time of ovulation. During menstruation, it is often associated with dysmenorrhea and before or during menstruation, migraine is frequently refractory to treatment. These are the times of greatest fluctuation in estrogen levels. The primary trigger of menstrual migraine appears to be the withdrawal of estrogen rather than progesterone. Changes in the sustained estrogen levels with pregnancy (increased) and menopause (decreased) can result in changes in headache frequency and intensity. Women who have migraine exclusively with their menses can be treated by the perimenstrual use of prophylactic medication (antidepressants, beta- 